\title{
Network and Economic Trade-Off Performance Regions of Cognitive Radio Systems with Power Control
}

\author{
Ramiro Sámano-Robles $^{1, *}$ and Atílio Gameiro ${ }^{2}$ \\ ${ }^{1}$ Instituto de Telecomunicações, Campus Universitário, Aveiro, 3810-193, Portugal \\ ${ }^{2}$ Departamento de Electronica, Universidade de Aveiro, Aveiro, Portugal
}

Received: 5 Jan. 2013, Revised: 7 May 2013, Accepted: 9 May 2013

Published online: 1 Sep. 2013

\begin{abstract}
Cognitive radio will enable terminals with access to licensed and unlicensed portions of the spectrum. This feature is expected to solve bandwidth scarcity problems in future wireless networks. However, different parts of the spectrum will be subject not only to different propagation conditions, but also to different licensing and billing agreements. Therefore, in order to obtain the major profit and spectrum efficiency, resource allocation algorithms must now target both network and economic performance metrics. This problem can be conveniently expressed as a multi-objective portfolio optimization problem, which has been studied in detail in the field of economics. This paper addresses the study of network and economic Pareto optimal trade-off performance regions of cognitive radio systems under average transmit power control policies. Each packet transmission in primary and secondary mode is regarded as a financial asset whose average transmit power is optimized so as to simultaneously maximize return and minimize risk, where risk is the variance of the return. This paper studies three types of Pareto optimal trade-off regions: primary vs. secondary throughput, return vs. risk, and sum-throughput vs. fairness, where fairness is evaluated by means of the Gini index. The boundaries of these trade-off regions are derived in parametric closed-form expressions. A power control policy is further proposed that maximizes return while simultaneously controlling risk and ensuring a level of quality of service for primary and secondary users. This means that operators can maximize revenue and network efficiency, while simultaneously minimizing risk and also ensuring fairness between primary and secondary users.
\end{abstract}

Keywords: Cognitive radio, multi-objective portfolio optimization, spectrum aggregation/selection.

\section{Introduction}

\subsection{Cognitive Radio (background)}

The increasing demand for higher data rates means that larger amounts of bandwidth are required to accommodate all traffic requests. Unfortunately, the current spectrum licensing paradigm has proved highly inefficient, as most of the licensed spectrum bands remain largely underutilized for long periods of time. This issue has led to spectrum scarcity problems for future applications [1].

A solution to this problem can be found in the area of cognitive radio, where smart, highly adaptive and efficient sensing and radio transmission devices are expected to opportunistically access different frequency bands without significantly disturbing licensed or primary users [2]- [4].

\subsection{Motivation}

Cognitive radio promises an adaptive and opportunistic access to different portions of the spectrum, thereby improving end-user satisfaction and partially solving bandwidth scarcity problems. However, different parts of the spectrum will be subject not only to different propagation and load conditions, but also to different licensing and billing agreements. For example, consider the terminal licensed to operate in LTE (long term evolution) in Fig. 1. With the help of cognitive radio, this terminal can opportunistically access an unlicensed frequency band such as the ISM band (industrial, scientific, and medic), which is commonly used for WiFi. Whenever the LTE service is degraded due to load or fading, and the ISM band is sensed as available, then the user can be handed over to WiFi without losing connectivity.

\footnotetext{
*Corresponding author e-mail: ramiro@av.it.pt
} 


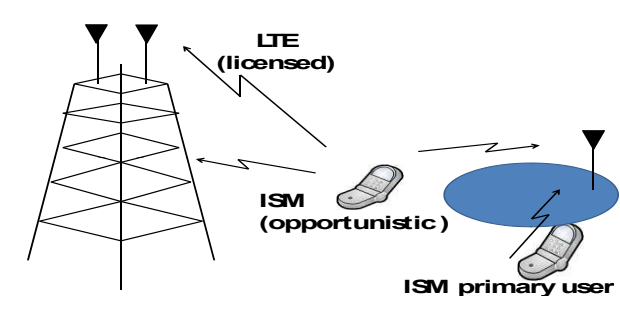

Fig. 1 Example of opportunistic spectrum access with cognitive radio.

In LTE, radio resources are usually allocated by the central scheduler in a dedicated fashion, thereby ensuring a good level of quality of service. By contrast, in the ISM band the availability of such resources is less reliable due to the contention process with all other users in contiguous WiFi networks or other ISM services (e.g., Bluetooth).

Regarding the billing of services, in the licensed LTE band the user can be charged according to the amount of data exchanged over the connection. This is because the operator has invested a considerable amount of money in spectrum licences and needs a return for its investment as well as a margin profit. By contrast, in the ISM band there are no fees for spectrum licenses. Therefore, if the connection is by means of WiFi, the user can be charged in terms of the duration of the connection with a fee relatively lower than in LTE. Conversely, it is also possible that the LTE operator does not owe ISM transmission technology, but instead it is provided by a third party operator that charges for hot-spot services over different geographical areas. In this case, the LTE operator might charge users with an additional fee for opportunistic services in the ISM band to cover for the cost of the third-party networking services.

As it can be observed with this simple example, the licensing and billing agreements of different portions of spectrum, as well as their radio resource management requirements can be considerably different and complex. In this heterogeneous landscape, operators face the following problems with cognitive radio networks:

1.How to allocate spectrum resources so as to obtain the maximum network and spectral efficiencies?

2.How to allocate spectrum resources in order to maximize the return of investment and margin profit, while simultaneously reducing risk or variations of the return?, and

3.How to bill the users for the licensed and opportunistic services so that the prices are competitive and attractive in the market and the return and network resource efficiency are kept at an acceptable level?

This paper aims to partially answer these questions by merging economic and networking optimization tools in the analysis of cognitive radio networks with average transmit power control.

\subsection{Paper contributions}

The allocation of radio resources in a cognitive radio network with heterogeneous licensing/billing agreements, as well as different network and radio propagation conditions must reflect the complex trade-off between the network and economic worlds. This problem can be conveniently expressed as a multi-objective portfolio optimization problem, which has been investigated in detail in the fields of economics and finance theory [5]- [8]. This paper attempts to partially address this issue by optimizing the transmit power for primary and secondary transmissions in terms of economic indicators, such as return and risk, in addition to the conventional network performance metrics such as throughput and load.

This paper proposes a multi-objective function that attempts to optimize the average transmit power on each available resource of each frequency band such that the total return is maximized and the risk (or variance of the return) is minimized or controlled. This economic-optimum average transmit power policy is compared to the policy that maximizes the throughput region of the system. In this work, it is assumed that both primary and secondary transmissions transmit simultaneously all the time, thereby interfering with each other. Our aim is to explore spatial reuse in the context of cognitive radio. The extension to more complex multiple access and cognitive sensing schemes is straightforward. To facilitate analysis, a packet reception model for Rayleigh channels is also proposed that allows for the calculation of correct packet reception statistics in closed-form expressions in the presence of interference between primary and secondary users.

The boundaries of three types of Pareto optimal trade-off performance regions are here derived in parametric closed-form expressions: primary vs. secondary throughput, risk vs. return, and sum throughput vs fairness. By inspection and by analytical work, conditions are derived for simultaneously achieving maximum throughput and maximum economic performance. This means that operators can simultaneously maximize revenue and network resource efficiency. A transmit power control policy is further proposed that maximizes return while, simultaneously, controlling risk and ensuring a level of quality of service for primary and secondary users. This means that operators can maximize economic and network efficiency while ensuring a given level of fairness between primary and secondary users. The trade-off performance regions of this transmission policy are characterized and discussed. The results in this paper also give some indication of the economic parameters in terms of return and risk that will provide operators with optimum network and revenue performances, which can be also useful in the design of billing schemes. 


\subsection{Related works and comparison}

Techno-economic analysis and study of wireless networks has been addressed before in the literature. The conventional approach was the use of a techno-economic model to evaluate the revenue of an operator under a given set of resource allocation assumptions. The main objective was to find the optimum resource allocation that provides the largest revenue and that satisfies users of the network [9]. In the context of cognitive radio, research in this area has been intensive due to the relevance of understanding the potential gains of opportunistic spectrum usage. A review of different approaches for the use of economic optimization tools in cognitive radio can be found in [10], where the authors have proposed a market equilibrium approach where primary and secondary users implement a learning algorithm so that they can adapt the amount of spectrum used, the pricing and the optimum demand that achieve equilibrium. Most of the existing works are based on game theoretic concepts (see [11]- [15]). The work in [14] has used an atomic congestion game theoretic approach in a wireless network with spatial reuse and inter-user interference. The work in [15] addresses the problem of calculating the optimum spectrum pricing in a dynamic market spectrum. Another related approach for the use of economics in cognitive radio can be found in works such as [16] and [17] and references therein, which are based on the concepts of auction theory.

This paper addresses the optimization of cognitive radio networks under a slightly different approach. We use multi-objective portfolio optimization under the assumption that each packet transmission is a financial asset. Our work explicitly introduces the concept of risk in the resource allocation problem and derives relevant expressions that allow for an interpretation of the resource allocation problem as a financial stock market problem. The work in [18] has used the concept of return and variance of the return but in the context of spectrum pricing and copyright. The work in [7] has used a multi-objective criteria optimization in the problem of radio access technology selection. Our approach is different from these previous works regarding the explicit use of multi-objective optimization and the exploration of the boundaries of different Pareto optimal trade-off regions. This allows us to visualize geometrical attributes and the potential trade-off between network and economic performance metrics. In other words, instead of deriving a resource allocation policy that achieves a Nash or market equilibrium between primary and secondary users as in previous works, here we explicitly explore the boundaries of different trade-off performance regions, and from their study we propose a policy that achieves a good trade-off in terms of network and economic performance. In this sense, our approach complements previous works in the literature by providing a framework for trade-off analysis and explicit interpretation of financial market stock tools in cognitive wireless networks.
This paper is organized as follows. Section 2 describes the system and signal models. Section 3 presents the packet reception model for primary and secondary user transmissions. Section 4 describes the performance metrics (network and economic) including the definition of throughput, return vs. risk and sum-throughput vs. fairness trade-off performance regions. Section 5 proposes the transmission policies that achieve the boundaries of the three types of trade-off performance region, as well as an additional policy that ensures a level of fairness between primary and secondary users. Section 6 presents the results by means of sketches of the three types of region under different network and economic conditions, and finally Section 7 presents the conclusions.

\section{System and signal model}

Consider the deployment scenario in Fig. 2 with $L=2$ networks, operating in $K=2$ different frequency bands $f_{1}$ and $f_{2}$, respectively. The two networks are assumed to have a total of $M$ orthogonal radio resources available for allocation on each of the frequency bands, one for primary (licensed) transmissions, and the other one for secondary (opportunistic) transmissions. Network $l=1$ uses frequency $f_{1}$ for licensed transmissions and $f_{2}$ for opportunistic transmissions. By contrast, network $l=2$ uses frequency $f_{2}$ for licensed and $f_{1}$ for secondary transmissions. For simplicity, we consider all the resources to be statistically identical.

Let us focus on a single radio resource: a primary user will experience a channel with its serving base station denoted by $h_{p}$ and a channel with the potential source of interference given by a secondary transmission denoted by $h_{s p}$. Similarly, a secondary user will experience a channel with its serving base station denoted by $h_{s}$ and a channel with the potential source of interference from a primary transmission denoted by $h_{p s}$. All channels will be modelled as circular complex Gaussian random variables with zero mean and variances denoted, respectively, by: $\sigma_{p}^{2}, \sigma_{s}^{2}, \sigma_{s p}^{2}$, and $\sigma_{p s}^{2}$. It is assumed that both BSs always have information ready to be transmitted to the two types of user (full queue), which means that they permanently interfere with each other. The extension to more complex medium access control schemes with interference or collision avoidance is straightforward. Each BS attempts to control the transmit power of primary and secondary transmissions based on the information collected during the sensing and cognitive phases. The aim of this power control scheme is to achieve a good trade-off performance in terms of different metrics that will be defined later in this paper.

Now, consider that primary and secondary users receive packets with $N$ symbols, denoted here, respectively, by $\mathbf{x}_{p}=\left[x_{p}(0), \ldots, x_{p}(N-1)\right]^{T}, \quad$ and $\mathbf{x}_{s}=\left[x_{s}(0), \ldots, x_{s}(N-1)\right]^{T}$, where $(\cdot)^{T}$ is the vector transpose operator. Let us assume that packets are 
transmitted with average transmit power values given by: $E\left[\mathbf{x}_{p}^{H} \mathbf{x}_{p}\right]=r_{p}$ and $E\left[\mathbf{x}_{s}^{H} \mathbf{x}_{s}\right]=r_{s}$, where $E[\cdot]$ is the statistical average operator. The signals of primary and secondary transmissions in the presence of permanent interference are thus given, respectively, by:

$\mathbf{y}_{p}=h_{p} \mathbf{x}_{p}+h_{s p} \mathbf{x}_{s}+\mathbf{v}_{p}, \quad$ and $\quad \mathbf{y}_{s \mid p}=h_{s} \mathbf{x}_{s}+h_{p s} \mathbf{x}_{s}+\mathbf{v}_{s}$,

where $\quad \mathbf{v}_{p}=\left[v_{p}(0), \ldots, v_{p}(N-1)\right]^{T} \quad$ and $\mathbf{v}_{s}=\left[v_{s}(0), \ldots, v_{s}(N-1)\right]^{T}$ are the Gaussian noise vectors modelled as a complex circular Gaussian variables with zero mean and variance $\sigma_{v}^{2}: v(n) \sim \mathscr{C} \mathscr{N}\left(0, \sigma_{v}^{2}\right)$. The instantaneous signal-to-interference-plus-noise ratio (SINR) for primary and secondary transmissions in the presence of interference can be written, respectively, as:

$$
\gamma_{p}=\frac{r_{p}\left|h_{p}\right|^{2}}{\sigma_{v}^{2}+r_{s}\left|h_{s p}\right|^{2}}, \quad \text { and } \quad \gamma_{s}=\frac{r_{s}\left|h_{s}\right|^{2}}{\sigma_{v}^{2}+r_{p}\left|h_{p s}\right|^{2}}
$$

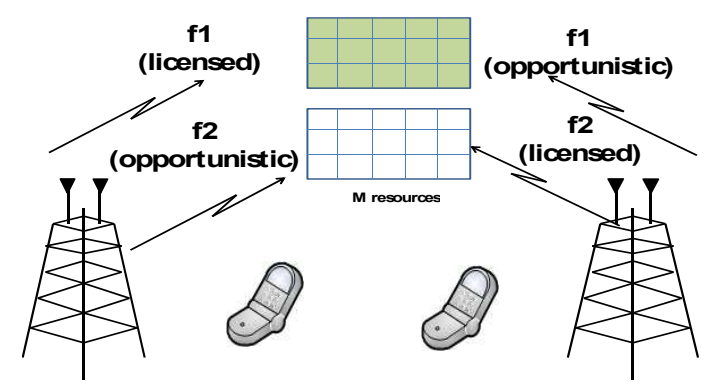

Fig. 2 System scenario for cognitive radio.

\section{Packet reception model}

This section presents the packet reception model for primary and secondary transmissions. The correct reception probabilities of packet transmissions towards primary and secondary users in the presence of interference, denoted here, respectively, by $q_{p}$ and $q_{s}$, are defined as the probability that the instantaneous SINRs in (2) surpass a reception threshold $\beta$ :

$$
q_{p}=\operatorname{Pr}\left\{\gamma_{p}>\beta\right\} \quad \text { and } \quad q_{s}=\operatorname{Pr}\left\{\gamma_{s}>\beta\right\} .
$$

By substituting (2) in the previous expression for the correct reception probability $q_{p}$, we obtain:

$$
q_{p}=\operatorname{Pr}\left\{r_{p}\left|h_{p}\right|^{2} /\left(\sigma_{v}^{2}+r_{s}\left|h_{s p}\right|^{2}\right)>\beta\right\},
$$

which can be rewritten as follows

$$
q_{p}=\operatorname{Pr}\left\{r_{p}\left|h_{p}\right|^{2}>\beta\left(\sigma_{v}^{2}+\left|h_{s p}\right|^{2}\right)\right\}
$$

$$
=\operatorname{Pr}\left\{r_{p}\left|h_{p}\right|^{2}-\beta r_{s}\left|h_{s p}\right|^{2}>\beta \sigma_{v}^{2}\right\} .
$$

By using the change of variables $z_{p}=r_{p}\left|h_{p}\right|^{2}$, $u_{p}=-\beta r_{s}\left|h_{s p}\right|^{2}$, and $w_{p}=z_{p}+u_{p}$, then (4) becomes:

$$
q_{p}=\operatorname{Pr}\left\{z_{p}+u_{p}>\beta \sigma_{v}^{2}\right\}=\operatorname{Pr}\left\{w_{p}>\beta \sigma_{v}^{2}\right\} .
$$

Therefore, it is now possible to calculate the statistics of $w_{p}$ to derive an analytical expression for $q_{p}$. Let us first consider that the probability density and the characteristic functions of $z_{p}$ and $u_{p}$ are given, respectively, by

$f_{z_{p}}\left(z_{p}\right)=\frac{1}{\widehat{z}_{p}} e^{-z_{p} / \widehat{z}_{p}}, \quad \Psi_{z_{p}}(i \omega)=\left(1-i \omega \widehat{z}_{p}\right)^{-1}, \quad z_{p}>0$,

where $\widehat{z}_{p}=E\left[z_{p}\right]=r_{p} \sigma_{p}^{2}$, and

$f_{u_{p}}\left(u_{p}\right)=\frac{1}{\widehat{u}_{p}} e^{u_{p} / \widehat{u}_{p}}, \quad \Psi_{u_{p}}(i \omega)=\left(1+i \omega \widehat{u}_{p}\right)^{-1}, \quad u_{p}<0$,

where $\widehat{u}_{p}=E\left[u_{p}\right]=\beta r_{s} \sigma_{s p}^{2}$. Since $z_{p}$ and $u_{p}$ are statistically independent, the characteristic function of their sum $w_{p}=z_{p}+u_{p}$ is given by the product of their individual characteristic functions in (6) and (7):

$\Psi_{w_{p}}(i \omega)=\Psi_{z_{p}}(i \omega) \Psi_{u_{p}}(i \omega)=\left(1-i \omega \widehat{z}_{p}\right)^{-1}\left(1+i \omega \widehat{u}_{p}\right)^{-1}$

which can be rewritten by partial fraction expansion (PFE) as:

$$
\Psi_{w_{p}}(i \omega)=A_{p}\left(1-i \omega \widehat{z}_{p}\right)^{-1}+B_{p}\left(1+i \omega \widehat{u}_{p}\right)^{-1},
$$

where $A_{p}=\left(1+\widehat{u}_{p} / \widehat{z}_{p}\right)^{-1}$ and $B_{p}=\left(1+\widehat{z}_{p} / \widehat{u}_{p}\right)^{-1}$. The back-transform of (9) provides a CCDF of $w_{p}$ given by:

$$
1-F_{w_{p}}\left(w_{p}\right)=A_{p} e^{-w_{p} / \widehat{z}_{p}} .
$$

Finally, the correct reception probability of a primary transmission in the presence of interference from a secondary user can be calculated as the CCDF in (10) valued at $w_{p}=\beta \sigma_{v}^{2}$ :

$$
q_{p}=1-F_{w_{p}}\left(\beta \sigma_{v}^{2}\right)=A_{p} e^{-\beta \sigma_{v}^{2} / \widehat{z}_{p}} .
$$

Following the lines of the derivation of (11), the expression for correct reception probability of a secondary transmission in the presence of interference from a primary user is given by $q_{s}=A_{s} e^{-\beta \sigma_{v}^{2} / \widehat{z}_{s}}$, where $A_{s}=\left(1+\widehat{u}_{s} / \widehat{z}_{s}\right)^{-1}, \widehat{z}_{s}=r_{s} \sigma_{s}^{2}$ and $\widehat{u}_{s}=\beta r_{p} \sigma_{p s}^{2}$.

\section{Trade-Off performance regions}

\subsection{Throughput region}

The main network performance metric to be used in this paper is packet throughput, which can be defined as the 
long-term ratio of total number of correctly transmitted packets to the total number of time-slots used in the measurement. The packet throughput of primary users can be related to the correct packet transmission probability as follows:

$$
T_{p}=(1-\eta) q_{p}
$$

where $\eta$ is the block error rate (BLER) of the modulation and coding scheme being used for the transmission, $q_{p}=\operatorname{Pr}\left\{\gamma_{p}>\beta\right\}$ is the packet reception probability in (11), and $\beta$ is the reception threshold for the given modulation and coding scheme and for the given BLER $\eta$. In this paper, we will assume all packet transmissions will use the same modulation and coding scheme and therefore for simplicity the throughput will be considered as equal to the reception probability:

$$
T_{p}=q_{p} \quad \text { and } \quad T_{s}=q_{s} .
$$

Let us now define the concept of throughput region following the concepts found in [19]. For this purpose, let $\mathbf{T}=\left[\begin{array}{ll}T_{p} & T_{s}\end{array}\right]^{T}$ be the vector of stacked throughput values of primary and secondary users, and $\mathbf{r}=\left[\begin{array}{ll}r_{p} & r_{s}\end{array}\right]^{T}$ be the vector of stacked average transmit power levels. The throughput region $\mathscr{C}_{T}$ is the union over all possible realizations of transmit power values:

$$
\mathscr{C}_{T}=\left\{\tilde{\mathbf{T}} \mid \tilde{T}_{p}=T_{p}(\mathbf{r}), \tilde{T}_{s}=T_{s}(\mathbf{r}), 0 \leq r_{p}, r_{s} \leq r_{\max }\right\},
$$

where $r_{\max }$ is the transmit power constraint. The throughput region can be also considered as the region where all the possible values of primary/secondary user throughput exist.

\subsection{Sum-throughput vs. fairness trade-off region}

The sum throughput of primary and secondary users can be simply defined as:

$$
T=T_{p}+T_{s}
$$

The metric for fairness to be used in this paper is the Gini index, which is commonly used in the field of economics. The Gini index takes value between zero and one: a value of one means that one user takes all the throughput (worst fairness) while the value of zero means complete fairness when all users get identical performances [21]. In our setting the fairness Gini indicator is defined as a minor variation of the definition in [21]:

$$
F_{G}=\frac{\left|T_{p}-T_{s}\right|}{T_{p}+T_{s}},
$$

where $|\cdot|$ is the absolute value operator. Let us now define the concept of sum-throughput vs. fairness trade-off region. For this purpose, let $\mathbf{F}=\left[\begin{array}{ll}T & F_{G}\end{array}\right]^{T}$ be the vector of stacked values of sum-throughput and fairness. The sum-throughput vs. fairness trade-off region $\mathscr{C}_{F}$ is the union over all possible realizations of transmit power values:

$$
\mathscr{C}_{F}=\left\{\tilde{\mathbf{F}} \mid \tilde{T}=T(\mathbf{r}), \tilde{F}=F(\mathbf{r}), 0 \leq r_{p}, r_{s} \leq r_{\max }\right\} .
$$

The sum-throughput vs. fairness trade-off region can be also considered as the region where all the possible values of sum-throughput and fairness exist.

\subsection{Return-risk region}

The main objective of this paper is to optimize the transmission parameters of primary and secondary users according to network and economic metrics. To achieve this goal, we will borrow concepts from the theory of multi-objective portfolio optimization, which is commonly used in the fields of economics and finance theory [6]. In a financial portfolio optimization problem, a portion of each financial asset from a given set of assets has to be optimized so as to maximize revenue or return and simultaneously control or minimize the risk or variance of the return [6]. In this paper, we will consider each correct packet transmission as a financial asset. Therefore, we will attempt to optimize the transmit power levels of primary and secondary transmissions in the same way as optimum allocation weights for the different assets are calculated in a financial portfolio optimization problem. For this purpose, let us define some economic metrics such as the instantaneous return per correctly transmitted packet in primary mode as $p_{p}$, and in secondary mode as $p_{s}$. Since packet reception is a random process, let us define the instantaneous return of a primary user as follows:

\section{Instantaneous return primary $=$}

$$
R_{p}=p_{p} t_{p}
$$

where $t_{p}$ is a binary random variable that takes the value of $t_{p}=1$ if the packet was correctly transmitted and the value of $t_{p}=0$ if the packet was incorrectly transmitted. By averaging the previous expression we obtain:

$$
\begin{gathered}
\text { Average return primary }=E\left[R_{p}\right] \\
=\hat{R}_{p}=E\left[p_{p}\right] \operatorname{Pr}\left\{t_{p}=1\right\}=\hat{p}_{p} \operatorname{Pr}\left\{\gamma_{p}>\beta\right\}=\hat{p}_{p} q_{p} .
\end{gathered}
$$

Following the lines of the previous derivation, the expressions for average return of secondary packet transmissions can be written as follows:

$$
\hat{R}_{s}=\hat{p}_{s} q_{s} \text {. }
$$

Let us now calculate the risk of a primary packet transmission as the variance of (18):

$$
\text { Risk primary }=S_{p}=E\left[\left(R_{p}-\hat{R}_{p}\right)^{2}\right]
$$




$$
\begin{gathered}
=E\left[R_{p}^{2}\right]-\hat{R}_{p}^{2}=E\left[p_{p}^{2}\right] \operatorname{Pr}\left\{t_{p}=1\right\}-\left(\hat{p}_{p} q_{p}\right)^{2}= \\
=E\left[p_{p}^{2}\right] \operatorname{Pr}\left\{\gamma_{p}>\beta\right\}-\left(\hat{p}_{p} q_{p}\right)^{2}=E\left[p_{p}^{2}\right] q_{p}-\left(\hat{p}_{p} q_{p}\right)^{2} .
\end{gathered}
$$

Note that the terms $E\left[p_{p}^{2}\right]$ and $E\left[p_{p}\right]=\hat{p}_{p}$ are related to the financial fluctuations of the transmissions in the primary band, whereas the terms $q_{p}$ and $q_{p}^{2}$ are related to the fluctuations of the channel conditions. Now, both types of fluctuations usually occur in different time-scales. Since we are interested in short-term fluctuations, particularly in the time scale of radio resource allocation schemes, it would be possible to ignore the terms related to financial fluctuations. However, in this paper these terms will not be dropped as they will be used for assigning different priority levels to primary and/or secondary transmissions. This will allow us to control in a better manner the proposed short-term radio resource allocation schemes. Following the lines of the derivation of (21), the expression for the risk of secondary packet transmissions is given by:

$$
\hat{S}_{s}=E\left[p_{s}^{2}\right] q_{s}-\left(\hat{p}_{s} q_{s}\right)^{2} .
$$

Finally, the total average return combining primary and secondary transmissions, denoted here by $\hat{R}$, can be calculated as:

Total average return $=\hat{R}$

$$
=\hat{R}_{p}+\hat{R}_{s}
$$

Following the lines of the derivation of the previous expression, the total risk, denoted here by $\hat{S}$, can be calculated as follows:

$$
\text { Total Risk }=\hat{S}=\hat{S}_{p}+\hat{S}_{s}
$$

Having defined the total average return and the total risk, let us now define the concept of return-risk region. For this purpose, let $\mathbf{M}=\left[\begin{array}{ll}\hat{R} & \hat{S}\end{array}\right]^{T}$ be the vector of stacked return and risk values. The return-risk region $\mathscr{C}_{M}$ is the union over all possible realizations of transmission power levels:

$$
\mathscr{C}_{M}=\left\{\tilde{\mathbf{M}} \mid \tilde{R}=\hat{R}(\mathbf{r}), \tilde{S}=\hat{S}(\mathbf{r}), 0 \leq r_{p}, r_{s} \leq r_{\max }\right\},
$$

which can be simply considered as the region where all the possible values of return and risk exist.

\section{Optimum power control policies}

\subsection{Throughput-region optimum}

To derive the boundaries or envelope of the throughput region, a multi-objective optimization is here proposed, where all $T$ 's can be simultaneously optimized:

$\mathbf{r}_{o p t}=\arg \max _{\mathbf{r}}\left[\begin{array}{lll}T_{p}, & T_{s}\end{array}\right]$ subject to $r_{p}, r_{s}<r_{\max }$.
This multi-objective optimization problem can be reformulated by using the method of scalarization [6]:

$$
\mathbf{r}_{\text {opt }}=\arg \max _{\mathbf{r}} T_{p}+\mu_{T} T_{s}, \quad \text { subject to } \quad r_{p}, r_{s}<r_{\max }
$$

where $\mu_{T}$ is the trade-off weight between primary and secondary throughput functions. By solving this problem for different values of $\mu_{T}$ it is possible to obtain the boundaries of the throughput region, which can also be regarded as the Pareto optimal trade-off curve of the multi-objective optimization problem [6]. Since the throughput expressions for both primary and secondary users increase monotonically with the transmit power levels, their global maxima are given when one or both power levels are set to be equal to the maximum transmit power constraint $r_{\max }$. Therefore, the boundaries of the throughput region are given by the following set of curves:

$$
\check{T}_{p}=T_{p}\left(r_{p}=r_{\max }, r_{s} \in\left\{0, r_{\max }\right\}\right)
$$

and

$$
\breve{T}_{s}=T_{s}\left(r_{p}=r_{\max }, r_{s} \in\left\{0, r_{\max }\right\}\right)
$$

for the case when $r_{p}=r_{\max }$, and

$$
\check{T}_{p}=T_{p}\left(r_{p}=\in\left\{0, r_{\max }\right\}, r_{s}=r_{\max }\right)
$$

and

$$
\check{T}_{s}=T_{s}\left(r_{p}=\in\left\{0, r_{\max }\right\}, r_{s}=r_{\max }\right)
$$

for the case when $r_{s}=r_{\max }$.

\subsection{Sum-throughput vs. fairness region optimum}

To derive the boundaries of the sum-throughput vs. fairness trade-off region, a multi-objective optimization scheme is here proposed, where both the sum-throughput and fairness can be simultaneously optimized:

$$
\mathbf{r}_{\text {opt }}=\arg \max _{\mathbf{r}}\left[\begin{array}{lllll}
T, & F_{G}
\end{array}\right] \text { s.t. } \quad r_{p}, r_{s}<r_{\max } .
$$

This multi-objective optimization problem can be reformulated by using the method of scalarization:

$\mathbf{r}_{\text {opt }}=\arg \max _{\mathbf{r}} T+\mu_{F} F_{G}, \quad$ subject to $\quad r_{p}, r_{s}<r_{\max }$

where $\mu_{F}$ is the trade-off weight between primary and secondary throughput functions. Since the throughput and fairness expressions increase monotonically with the transmit power levels, their global maxima are given when one or both power levels are set to be equal to the maximum transmit power constraint $r_{\max }$. To derive the boundaries of this trade-off region, let us focus on two extreme cases. The worst fairness performance with the highest value of sum throughput occurs when the user with best channel conditions is allocated with the maximum power constraint. By contrast, the best fairness performance with the highest sum-throughput occurs 
when both primary and secondary users achieve the same throughput given the user with the worst channel is allocated with the maximum transmit power constraint. The boundaries of the sum-throughput vs. fairness region can then be simply obtained by extending the performance metrics from these two extreme points and by changing the value of the power of the user that was not initially allocated with the maximum power constraint. Therefore, the boundaries of the sum-throughput vs. fairness trade-off region when the primary user experiences better channel conditions than the secondary user are given by the following set of curves:

$$
\check{T}=T\left(r_{p}=r_{\max }, r_{s} \in\left\{0, r_{\max }\right\}\right)
$$

and

$$
\check{F}_{G}=F_{G}\left(r_{p}=r_{\text {max }}, r_{s} \in\left\{0, r_{\max }\right\}\right)
$$

when $r_{p}=r_{\max }$, and

$$
\check{T}=T\left(r_{p} \in\left\{r_{p}^{*}, r_{\max }\right\}, r_{s}=r_{\max }\right)
$$

and

$$
\check{F}_{G}=F_{G}\left(r_{p} \in\left\{r_{p}^{*}, r_{\text {max }}\right\}, r_{s}=r_{\text {max }}\right)
$$

when $r_{s}=r_{\max }$. The term $r_{p} *$ denotes the value of power for which the throughput of primary and secondary users is identical given $r_{s}=r_{\max }$. Similar expressions can be derived for the case when the secondary user experiences better channel conditions than the primary user.

\subsection{Return-risk optimum: Financial Portfolio optimization}

To derive the boundaries of the return-risk region, a multi-objective portfolio optimization is here proposed, where both return and risk functions can be simultaneously optimized:

$$
\mathbf{r}_{o p t}=\arg \max _{\mathbf{r}}\left[\begin{array}{lll}
\hat{R}, & \hat{S}
\end{array}\right] \text { subject to } \quad r_{p}, r_{s}<r_{\max } .
$$

This multi-objective optimization problem can be reformulated by using the method of scalarization:

$$
\mathbf{r}_{\text {opt }}=\arg \max _{\mathbf{r}} \hat{R}-\mu_{r} \hat{S}, \quad \text { subject to } \quad r_{p}, r_{s}<r_{\max }
$$

where $\mu_{r}$ is the trade-off weight between return and risk functions. By differentiating the objective function with respect to $r_{p}$ and $r_{s}$ we obtain:

$$
\frac{\partial \hat{R}}{\partial r_{p}}+\mu_{r} \frac{\partial \hat{S}}{\partial r_{s}}=0
$$

and

$$
\frac{\partial \hat{R}}{\partial r_{s}}+\mu_{r} \frac{\partial \hat{S}}{\partial r_{s}}=0 .
$$

By obtaining the parameter $\mu_{r}$ from any of the equations and substituting it back in the other expression we obtain:

$$
\frac{\partial \hat{R}}{\partial r_{p}} \frac{\partial \hat{S}}{\partial r_{s}}-\frac{\partial \hat{S}}{\partial r_{s}} \frac{\partial \hat{R}}{\partial r_{s}}=0,
$$

which can be also expressed as:

$$
\left|\mathbf{J}_{e}\right|=\left|\left(\begin{array}{ll}
\frac{\partial \hat{R}}{\partial r_{p}} & \frac{\partial \hat{R}}{\partial r_{s}} \\
\frac{\partial \hat{S}}{\partial r_{p}} & \frac{\partial \hat{S}}{\partial r_{s}}
\end{array}\right)\right|=0,
$$

where $\mathbf{J}_{e}$ is the Jacobian matrix. Due to the complexity of this expression, the solution will be obtained by means of a numerical method. The solution for this expression combined with the expressions in (23) and (24) provide a parametric form (in terms of the power levels) of the boundary of the return-risk region. This solution is complemented by the boundary conditions which are obtained by setting the transmit power variables equal to the maximum power constraint $r_{\max }$ or equal to zero. Sketches of the different trade-off regions are provided in the following section.

\subsection{Optimum transmission policy with fairness}

In the previous subsections we addressed the derivation of the boundaries of the three types of trade-off region. However, it is possible that none of the points of such boundaries provide a convenient network and economic performance solution for a given operator. To address this issue, let us now derive a transmission policy that maximizes return while controlling the risk, but which is also able to ensure a given throughput performance for primary and secondary users. This can be expressed as:

$$
\begin{array}{r}
\mathbf{r}_{o p t}=\arg \max _{\mathbf{r}} \hat{R}, \quad \text { s.t. } \quad \hat{S} \leq \hat{S}_{0}, \\
T_{p} \geq \alpha_{p} q_{p}, \quad T_{s} \geq \alpha_{s} q_{s}, \quad r_{p}, r_{s}<r_{\max }
\end{array}
$$

where $\alpha_{p}$ and $\alpha_{s}$ are the constraints on primary and secondary throughput performance, respectively. In the following derivation we assume that the inequality constraints $T_{p} \geq \alpha_{p} q_{p}$ and $T_{s} \geq \alpha_{s} q_{s}$ can be simultaneously achieved. Since the return is an increasing function in terms of the average power levels of primary and secondary users, the solution that complies with all the constraints can be found by exploring all the intersections points between the different equality constraints and the boundary of the return-risk region $\left(\hat{S}=\hat{S}_{0}, \quad T_{p}=\alpha_{p} q_{p}, \quad T_{s}=\alpha_{s} q_{s}\right.$ and $\mathbf{b d}\left(\mathscr{C}_{M}\right)$, where $\operatorname{bd}\left(\mathscr{C}_{M}\right)$ indicates the boundary of the region $\left.\mathscr{C}_{M}\right)$ and select the intersection with the best return value.

\section{Results and discussion}

This section presents the sketches of the three types of trade-off performance region studied in this paper. The 
results will be divided in two cases: one where the interference between secondary and primary users is high, and the second one where this interference is relatively low. Primary users will be modelled with channel parameter of $\sigma_{p}^{2}=4$, while secondary users will use a parameter of $\sigma_{s}^{2}=2$. Interference parameters in the high interference scenario will be given by $\sigma_{s p}^{2}=10$ and $\sigma_{p s}^{2}=12$. Interference parameters in the low interference scenario will be given by $\sigma_{s p}^{2}=0.5$ and $\sigma_{p s}^{2}=0.25$. Reception threshold is set to a value of $\beta=1$. Regarding the economic parameters, the secondary transmission will always be considered with a higher financial risk value with $E\left[p_{s}^{2}\right]-\hat{p}_{s}^{2}=10$, while the risk in primary transmission will be fixed too to a value of $E\left[p_{p}^{2}\right]-\hat{p}_{p}^{2}=0.1$. In the case of the average return we will consider two cases: one where the return of the primary transmission is higher than the average return of the secondary transmission $\left(\hat{p}_{p}=5, \hat{p}_{s}=1\right)$, and the second case where secondary transmissions experience higher average return than primary transmissions $\left(\hat{p}_{p}=1\right.$, $\left.\hat{p}_{s}=5\right)$.

Fig. 3, 4 and 5 show, respectively, the throughput, return-risk, and sum throughput vs. fairness trade-off regions in a system with high interference and where return in the primary is higher than the return in the secondary. Note that the throughput region in fig. 3 is non-convex. All figures show the region projected by the proposed power control policy with fairness considering the constraints $\alpha_{p}=0.3, \alpha_{s}=0.1$, and $\hat{S}_{0}=7$. The sum throughput vs. fairness region in Fig. 4 shows that worst fairness performance, which is also the point of higher sum-throughput performance, occurs when primary user is allocated with the maximum power constraint $r_{\text {max }}$. By contrast, the point with the best fairness performance is given by the point where the secondary user is allocated with the maximum power, at the expense of sum-throughput reduction. In this figure it can be also observed that the proposed policy maintains a fairness below the value of 0.6 , thereby showing that it provides good fairness performance. In the return vs. risk trade-off region in Fig. 5 it can be observed that the maximum risk performance is given when the secondary user is allocated with full power. By contrast, the lowest risk performance, which gives also the highest return performance, is given when the primary user is allocated with full power. Since none of these performance points provide fairness, it can be observed that the proposed policy with fairness gives us a good trade-off between the achieved return and the risk associated with the allocation. Note that the boundaries of this trade-off region are given exclusively by boundary conditions with maximum or null power allocation.

Fig. 6, 7 and 8 show, respectively, the throughput, return-risk, and sum throughput vs. fairness trade-off regions in a system with high interference and where return in the primary is lower than the return in the secondary. While the throughput and sum throughput vs.

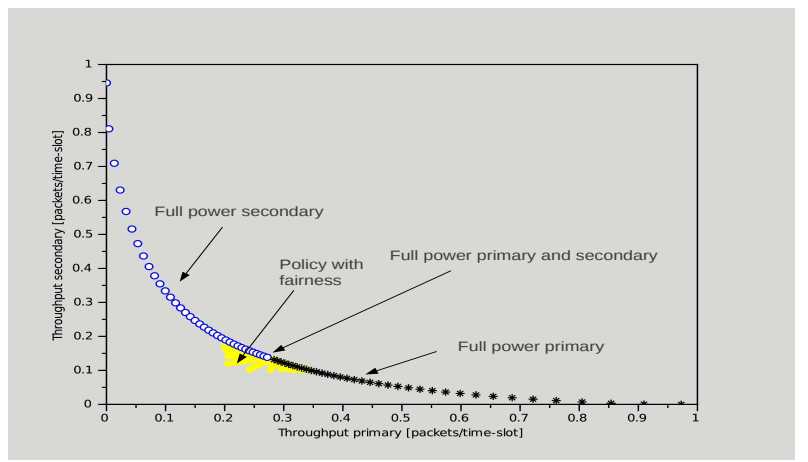

Fig. 3 Throughput region of a cognitive radio system with high interference and primary transmission with higher return than secondary transmission.

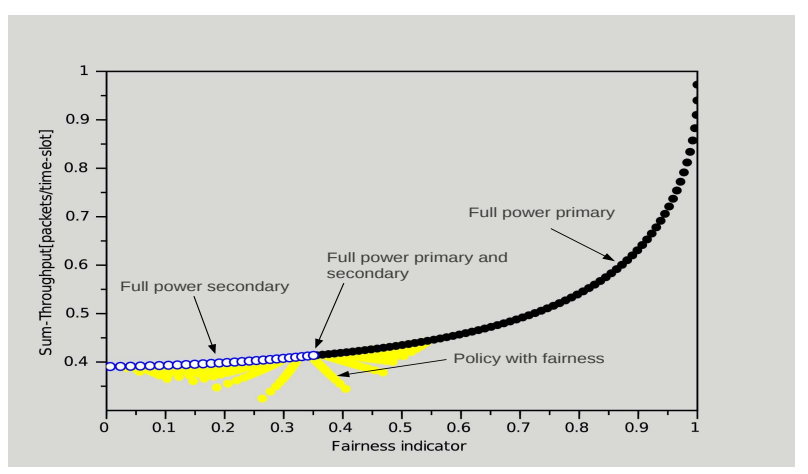

Fig. 4 Sum-throughput vs fairness trade-off region of a cognitive radio system with high interference and primary transmission with higher return than secondary transmission.

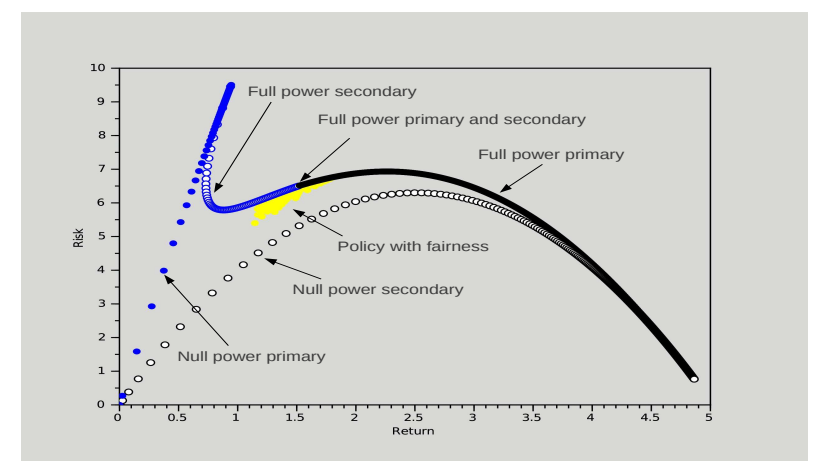

Fig. 5 Return-risk region of a cognitive radio system with high interference and primary transmission with higher return than secondary transmission. 
fairness trade-off regions remain quite similar to the previous case in Fig. 3 to Fig. 5, the return vs. risk trade-off region in Fig. 8 has been considerably changed. The point of maximum return is now given when the secondary user is allocated with full power, which also provides the point with maximum risk, as secondary transmissions have been considered as always more risky than primary transmissions. We can observe that in comparison with the previous example in Fig. 5 the return obtained by the policy with fairness is lower, and indeed the whole return performance seems to be much more limited. This suggests that having a primary system with higher return than a secondary system provides much more flexible allocation in order to obtain good performance both in terms of economic and network metrics.

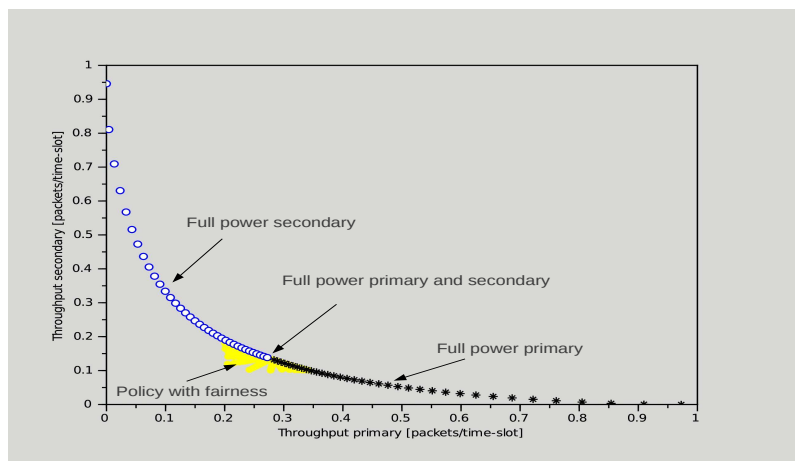

Fig. 6 Throughput region of a cognitive radio system with high interference and primary transmission with lower return than secondary transmission.

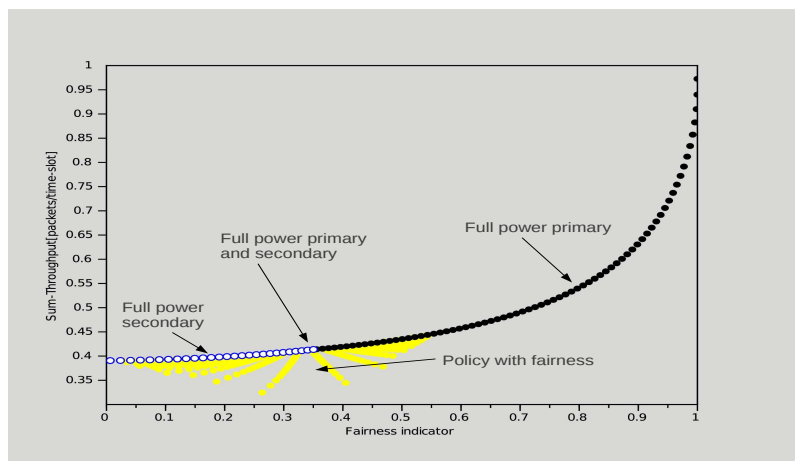

Fig. 7 Sum-throughput vs fairness trade-off region of a cognitive radio system with high interference and primary transmission with lower return than secondary transmission.

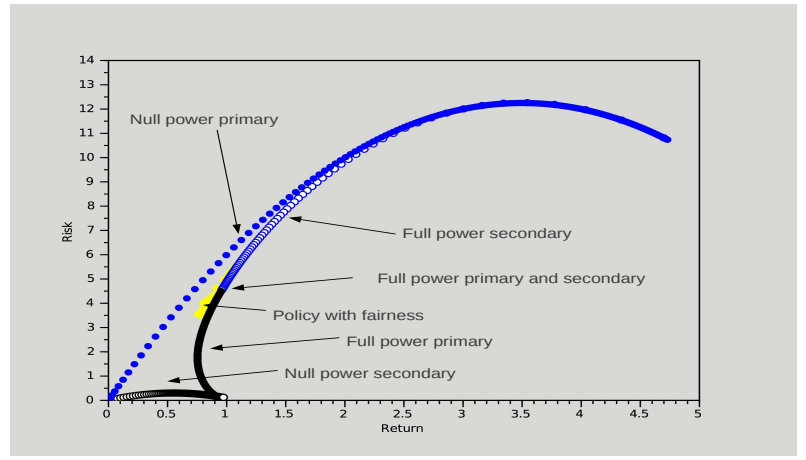

Fig. 8 Return-risk region of a cognitive radio system with high interference and primary transmission with lower return than secondary transmission.

Fig. 9, 10 and 11 show, respectively, the throughput, return-risk, and sum throughput vs. fairness trade-off regions in a system with low interference and where return in the primary is lower than the return in the secondary. It can be observed that the throughput region in Fig. 9 is now convex as compared to the case with high interference in Fig. 6 and 3. The sum-throughput vs. fairness trade-off region has also changed. The maximum sum throughput is now given by the point where the secondary user is allocated with full power and not the primary user as in the previous cases. The return vs. risk region in Fig. 11 is also different. In comparison with the previous cases, where the boundaries were given by simply using maximum or null power allocation conditions, in this case one part of the boundary is given by the solution of the jacobian determinant in eq.(40). The proposed policy with fairness can be observed in all figures to comply with the constraints on throughput and risk.

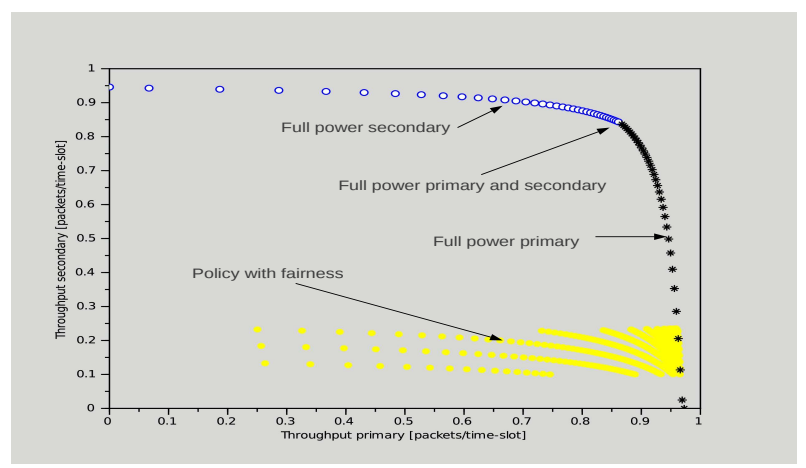

Fig. 9 Throughput region of a cognitive radio system with low interference and primary transmission with lower return than secondary transmission. 


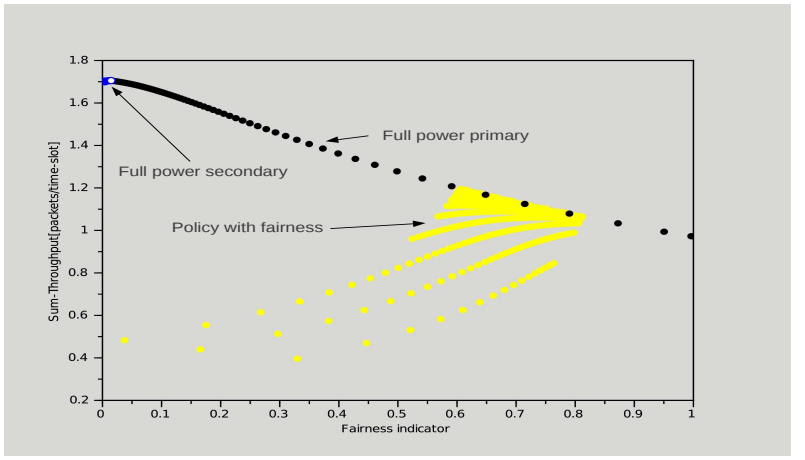

Fig. 10 Sum-throughput vs fairness trade-off region of a cognitive radio system with low interference and primary transmission with lower return than secondary transmission.

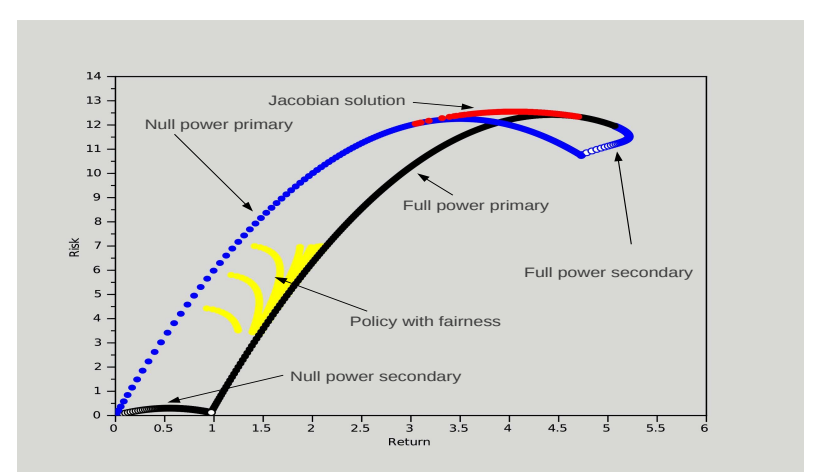

Fig. 11 Return-risk region of a cognitive radio system with low interference and primary transmission with lower return than secondary transmission.

Fig. 12, 13 and 14 show, respectively, the throughput, return-risk, and sum throughput vs. fairness trade-off regions in a system with low interference and where return in the primary is higher than the return in the secondary. The main difference with respect to the previous case is that the return-risk region in Fig. 14 is now larger than the region in the case where primary has lower return than the secondary, and mainly the proposed policy with fairness has a much better and extended performance over the projected region. This confirms the previous result where better economic performance is obtained when the return in the primary is higher than the return in the secondary. This result can be useful in the design of billing and pricing schemes that give more weight to primary than to secondary transmissions. This also suggests that in order to obtain a good economic performance, a given operator might want to keep a reliable licensed primary band to support the major part of the requested traffic, and a secondary band which is more unreliable, more risky and with lower return than the primary band in order to provide additional opportunistic services.

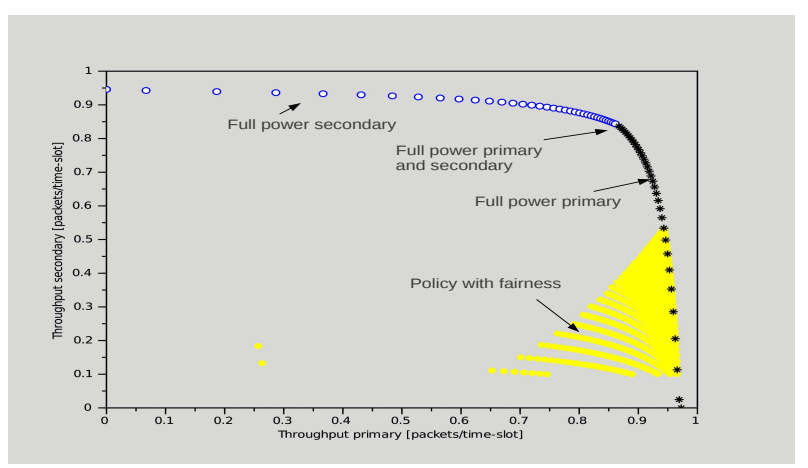

Fig. 12 Throughput region of a cognitive radio system with low interference and primary transmission with higher return than secondary transmission.

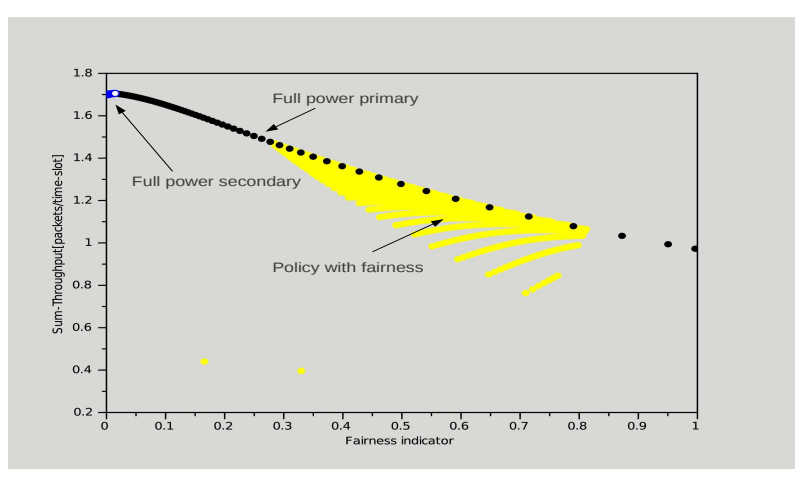

Fig. 13 Sum-throughput vs fairness trade-off region of a cognitive radio system with low interference and primary transmission with higher return than secondary transmission.

\section{Conclusions}

This paper has presented the derivation of the optimum average power transmission policies that will allow a cognitive radio system to achieve, respectively, the boundaries of three types of trade-off regions: primary versus secondary throughput performance, sum throughput vs. fairness, and return versus risk (variance of the return) trade-off performance. The later transmission policy is based on a multi-objective portfolio optimization approach that blends network with economic performance metrics. A packet reception model was also proposed for Rayleigh channels to derive closed-form expressions that 


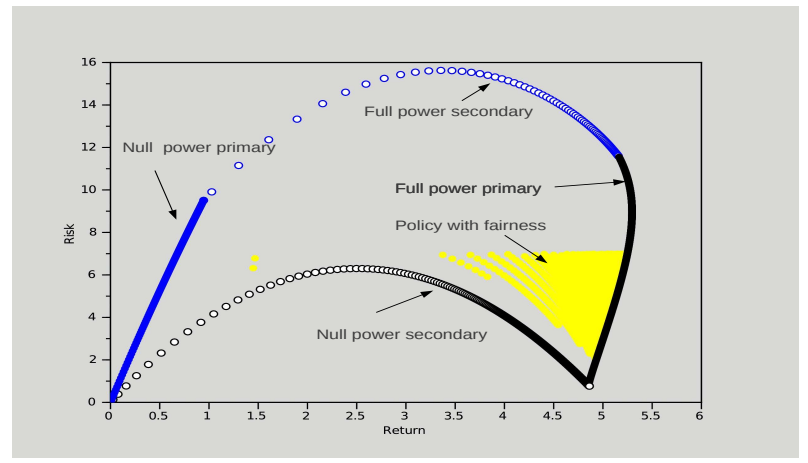

Fig. 14 Return-risk region of a cognitive radio system with low interference and primary transmission with higher return than secondary transmission.

can be included in all derivations. The boundaries of the regions were derived also in closed-form expression and sketches were further provided for scenarios with different interference assumptions and economic performance metrics. Particular good results were obtained when the return in the primary band is higher than the return in the secondary frequency band. In view that the boundaries of the two types of region tend to be unfair to primary or secondary users, a third transmission policy was proposed that allows to maximize return, control the risk and to ensure a certain level of quality of service for primary and secondary user transmissions. This means that operators will be able to maximize network and economic performance while ensuring fairness between the different types of users. The results can help operators improve network performance and to derive optimum billing schemes that will allow maximize revenue and quality of service simultaneously. the results in this paper have assumed continuous transmission of primary and secondary users. Future work includes the use of more advanced contention avoidance and medium access control schemes for cognitive radio systems.

\section{References}

[1] R. Pal, D. Idris, K. Pasari, and N. Prasad, "Characterizing Reliability in cognitive radio networks," First Int. Symposium on Applie Sciences on Biomedical and Commun. tech., 1-6 (2008),

[2] L. Lu, X. Zhou, U. Onunkwo, and G. Y. Li, "Ten years of research in spectrum sensing and sharing in cognitive radio," EURASIP Journal on Wireless Commun. and Networking, (2012).

[3] W. Jouini, C. Moy, and J. Palicot "Decision making for cognitive radio equipment: analysis of the first 10 years of exploration," EURASIP Journal on Wireless Commun. and Networking, (2012).
[4] J. Mitola Software radios: survey, critical evaluation and future directions IEEE Aerosp. Electron. Syst. Mag., 8, 2531, (1993).

[5] F. J. Fabozzi, et al., Robust Portfolio Optimization and Management Wiley Finance, (2007).

[6] S. Boyd and L. Vandenberghe, Convex optimization, Cambridge University Press, (2004).

[7] M. M. Alkhawlani, and A. T. Zahar, "Intelligent Radio Network Selection for Next Generation Networks" Journal of Intelligent Computing, 1, 64-74 (2010).

[8] A. Konak, D. W. Coit, and A. E. Smith, "Multi-objective optimization using genetic algorithms: A tutorial," Reliability Engineering and System Safety, Special issue in genetic algorithms and reliability, 91, 992-1007.

[9] T. Smura, "Techno-economic modelling of wireless network and industry architectures," Doctoral dissertation, Aalto University, (2012).

[10] D. Niyato and E. Hossain, "Spectrum trading in cognitive radio networks: A market-equilibrium-based approach," IEEE Wireless Communications, 15, 71-80 (2008).

[11] R. Southwell, X. Chen, and J. Huang, "Quality of Service Satisfaction Games for Spectrum Sharing," IEEE INFOCOM - Mini Conference. Turin, Italy, (2013).

[12] X. Chen, and J. Huang, "Spatial Spectrum Access Game: Nash Equilibria and Distributed Learning," ACM Mobihoc. Hilton Head Island, South Carolina, (2012).

[13] Duan, Lingjie, Jianwei Huang, and Biying Shou. "Duopoly Competition in Dynamic Spectrum Leasing and Pricing," IEEE Transactions on Mobile Computing, 11, 1706-1719 (2012).

[14] Tekin, Cem, et al. "Atomic Congestion Games on Graphs and Their Applications in Networking," IEEE Transactions on Networking, 20, 1541-1552 (2012).

[15] L. Duan, J. Huang, and B. Shou, "Investment and Pricing with Spectrum Uncertainty: A Cognitive Operator's Perspective," IEEE Transactions on Mobile Computing, 10, 1590-1604 (2011).

[16] Y. Zhang, D. Niyato, P. Wang, and E. Hossain, "Auctionbased resource allocation in cognitive radio systems," IEEE Communications Magazine, 50, 108-120 (2008).

[17] J. Huang, R. Berry, and M. L. Honig, "Auction-based Spectrum Sharing," Springer Journal Mobile Networks and Applications, 11, 405-408 (2006).

[18] T. A. Wysocki and A. Jamalipour, "An Economic Welfare Preserving Framework for Spot Pricing and Hedging of Spectrum Rights for Cognitive Radio," IEEE Transactions on Network and Service Management, 9, $87-99$ (2012).

[19] J. Luo and A. Ephremides "On the throughput, capacity, and stability regions of random multiple access," IEEE Transactions on Information Theory, 52, 2593-2607 (2006).

[20] R. Samano-Robles, M. Ghogho and D. C. McLernon "Wireless Networks with retransmission diversity and carrier sense multiple access," IEEE Transactions on Signal Processing, 57, 3722-3726 (2009).

[21] A. W. Marshall and I. Olkin, Inequalities: Theory of Majorization and its applications, Academic Press, (1979). 


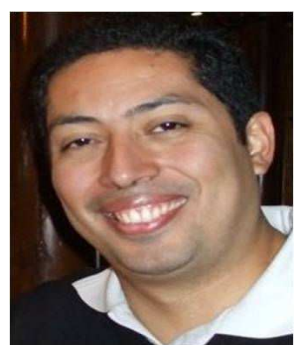

Ramiro Samano Robles received his Bachelor degree in Telecommunications in 2001 from the National Autonomous University of Mexico. In 2003, he received his MSc degree in Telecommunications and Information Systems from the University of Essex, UK, and the $\mathrm{PhD}$ degree in cross-layer design and signal processing for wireless networks from the University of Leeds in 2007. He currently holds a post-doctoral position at the Instituto de Telecomunicações in Aveiro, Portugal. His main interests lie in the areas of MAC-PHY cross-layer design, random access protocols, distributed antenna systems, radio frequency identification, and shaped reflectors design for satellite communications. He has over 20 technical papers in international journals and conferences. He also has professional experience in major telecommunication carriers in Mexico. He has been involved in several national and European projects, namely the CODIV, FUTON, ASPIRE, UNITE, CADWIN, and QoSMOS.

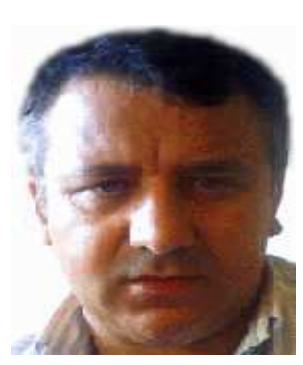

Atilio Gameiro received his Licenciatura (five years course) and his $\mathrm{PhD}$ from the University of Aveiro in 1985 and 1993 respectively. $\mathrm{He}$ is currently a Professor in the Department of Electronics and Telecom. of the University of Aveiro, and a researcher at the Instituto de Telecomunicações, Polo de Aveiro, where he is head of group. His industrial experience includes a period of one year at BT Labs and one year at NKT Elektronik. His main interests lie in signal processing techniques for digital communications and communication protocols, and within this research line he has done work for optical and mobile communications, either at the theoretical and experimental level, and has published over 120 technical papers in International Journals and conferences. His current research activities involve space-time-frequency algorithms for the broadband wireless systems and cross-layer design. He has been involved and has leaded IT or Univ of Aveiro participation in several national and European projects, namely the RACE projects MULTIGIGABIT, SPEED, MODAL , the ACTS project FRANS, and the IST projects ASILUM, MATRICE, 4MORE, ORACLE. He has coordinated CODIV and performed the technical management of the IP FUTON. 